\title{
The outcome of potassium dichromate exposure on histological structure of male rat sexual organs in suckling period
}

\author{
Jelena SAVICI ${ }^{1 *}$, Diana BREZOVAN ${ }^{1}$, Camelia TULCAN $^{1}$, Oana-Maria BOLDURA ${ }^{1}$ \\ ${ }^{1}$ Faculty of Veterinary Medicine, Banat's University of Agricultural Sciences and Veterinary Medicine King \\ Michael I of Romania Timisoara, Calea Aradului no. 119, 300654, Timisoara \\ *corresponding author: jelenarankov@yahoo.com
}

Bulletin UASVM Veterinary Medicine 77(1)/2020

Print ISSN 1843-5270; Electronic ISSN 1843-5378

doi:10.15835/buasvmcn-vm: 2019.0033

\begin{abstract}
The aim of this study was the evaluation of integrity biomarkers of reproductive toxicity: histoarchitecture of genital organs (testis and epididymis) and sexual accessory glands (prostate, seminal vesicles and bulbo-urethral glands) at sexual maturity after exposure to potassium dichromate (Cr VI) in suckling period. Consequent to the exposure of male rat pups to hexavalent chromium during suckling period structural changes appeared in genital organs and sexual accessory glands, such as: basal membrane and epithelial disintegration, interstitial edema, wavy basal membrane due to a reduction in tubule diameter, Leydig cell necrosis; epithelial smoothing, epithelial necrosis; epithelial cells desquamation and falling in lumen. Exposure to potassium dichromate (Cr VI) during suckling period produced severe congestive and degenerative lesions in genital organs and sexual accessory glands.
\end{abstract}

Keywords: chromium VI, histology, male, rat

\section{Introduction}

Manny heavy metals are known to affect testicular structure, spermatogenesis and steroidogenesis in man and animals, leading to infertility (Aruldhas et al., 2004).

Chromium is found in many compounds of the Earth's crust (Stoecker, 2004). It exists in several oxidation states, but Cr III and Cr VI species are the most stable and common forms (O’Brien et al., 2003).

Chromium III is essential for human and animal diet, it is poor soluble, with little or no toxicity. In its hexavalent form chromium is 100 to 1000 times more toxic than the most trivalent compounds (Stoecker, 2004).

Effluents from workplaces and industries contaminate the environment affecting man and animals living in those areas (Aruldhas et al.,
2004). The main sources of hexavalent chromium are electroplating process, stainless steel welding and vulcanizing industries, tanneries (ATSDR, 2012).

The aim of this study was the evaluation of $\mathrm{Cr}$ VI exposure impact on some male reproductive biomarkers. The objective was evaluation of chromium level and histoarchitecture of genital organs (testis and epididymis) and sexual accessory glands (prostate, seminal vesicles and bulbo-urethral glands).

\section{Materials and methods}

The study has been carried out, initially, on eight white Wistar female rats, which were obtained from authorized Biobase of University of Medicine and Pharmacy "Victor Babes" Timisoara, Romania. All rats were housed in standard 
polycarbonate cages in standard laboratory environmental conditions: temperature $22 \pm 2^{\circ} \mathrm{C}$, humidity $55 \pm 10 \%$, light/dark cycle $12: 12 \mathrm{~h}$. The animals were fed on standardized normal diet and water ad libitum. All the animals were handled in accordance with Directive 2010/63/EU and guideline of National Research Council. The Ethical Committee of Banat University of Agricultural Sciences and Veterinary Medicine Timișoara approved the experimental protocol.

Female rats, divided in four groups: three experimental and one control, were exposed via drinking water to potassium dichromate after delivery as follows: $\mathrm{E}_{1}$ - 25 ppm Cr VI (LOAEL - EPA,
1998); $\mathrm{E}_{2}$ - 50 ppm Cr VI; $\mathrm{E}_{3}$ - 75 ppm Cr VI, control received tap water without chromium content. During mating and gestation, females received only tap water. The pups from each experimental group were exposed via milk and drinking water. After weaning and until sexual maturity pups received only tap water without chromium.

At sexual maturity seven male rats from each group, experimental and control, were sacrificed following protocols and ethical procedures (ketamine and xylazine), and samples were collected. For histological assessment were used samples from genital organs and sexual accessory glands which were fixed in ethanol $80^{\circ}$ and

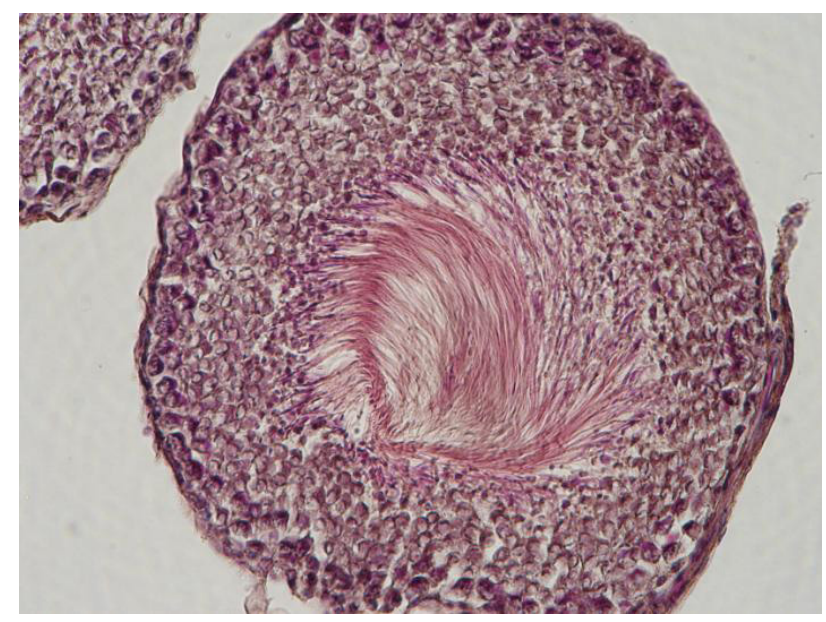

Figure 1. Histological section of testis from $\mathrm{E}_{1}$ group, Hematoxylin-Eosin stain, 400x a) basal membrane and epithelial desintegration, b) interstitial edema,

c) wavy basal membrane due to a reduction in tubule diameter

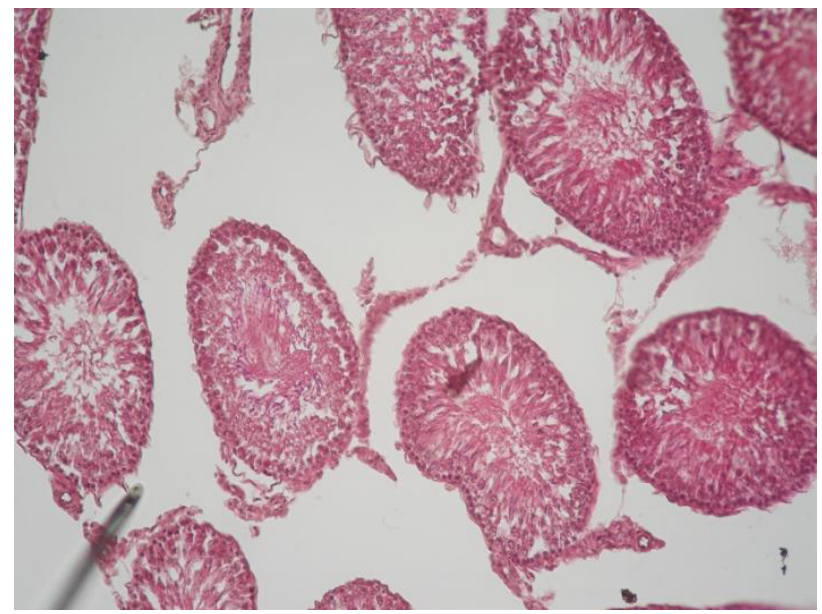

Figure 2. Histological section of testis from $\mathrm{E}_{3}$ group, Hematoxylin-Eosin stain, $200 \mathrm{x}$ a) basal membrane and epithelium degenerative-necrotic lesions, b) interstitial edema, c) Leydig cell necrosis 
embedded in paraffin wax. Then the embedded samples were cut using microtome Cut 4062 Slee Mainz, Germany in order to obtain histological sections of 5-micron thickness. The slides were stained by Hematoxylin and Eosin method, and then examined on Olympus microscope CX 41 provided with Olympus digital photo camera and image analysis software QuickPHOTO Micro 2.2.

\section{Results and discussions}

In all three experimental groups, exposed to hexavalent chromium, histoarchitecture of the genital organs and sexual accessory glands was altered. Namely, the lesions were: in testis interstitial edema, wavy basal membrane due to a reduction in tubule diameter, basal membrane and epithelium degenerative-necrotic lesions, Leydig cell necrosis (Fig. 1, 2); in epididymis interstitial edema, epithelial necrosis and exfoliation, epithelial smoothing (Fig. 3, 4); in prostate, seminal vesicles and bulbo-urethral glands epithelial desquamation and falling in lumen, epithelial segmental necrosis, flattening of the epithelium, fibrinous exudate (Figures 5, 6, 7).

Permeabilization of the testicular barrier and the accumulation of chromium in the testes could explain the occurred structural alterations (Chandra et al., 2007).

Chromium accumulation in the genital organs following exposure is also possible because

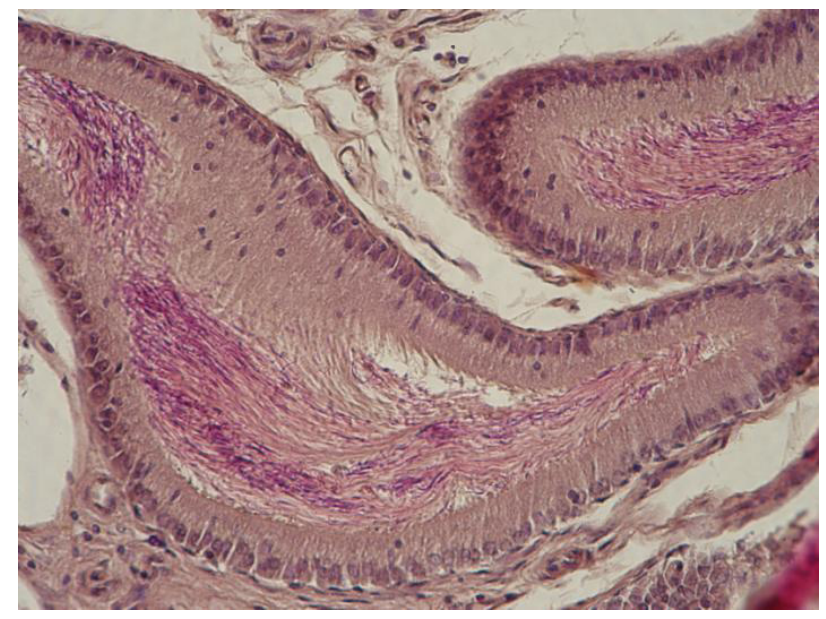

Figure 3. Histological section of epididymis from $\mathrm{E}_{2}$ group, Hematoxylin-Eosin stain, 400x $\leftarrow$ epithelial exfoliation

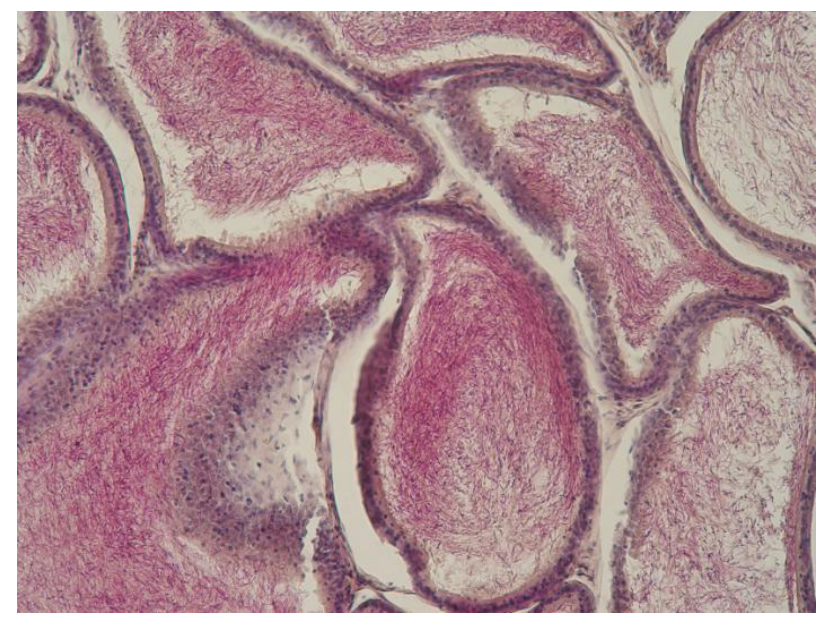

Figure 4. Histological section of epididymis from $\mathrm{E}_{3}$ group, Hematoxylin-Eosin stain, 200x a) epithelial exfoliation, b) flattening of the epithelium, c) epithelial necrosis 


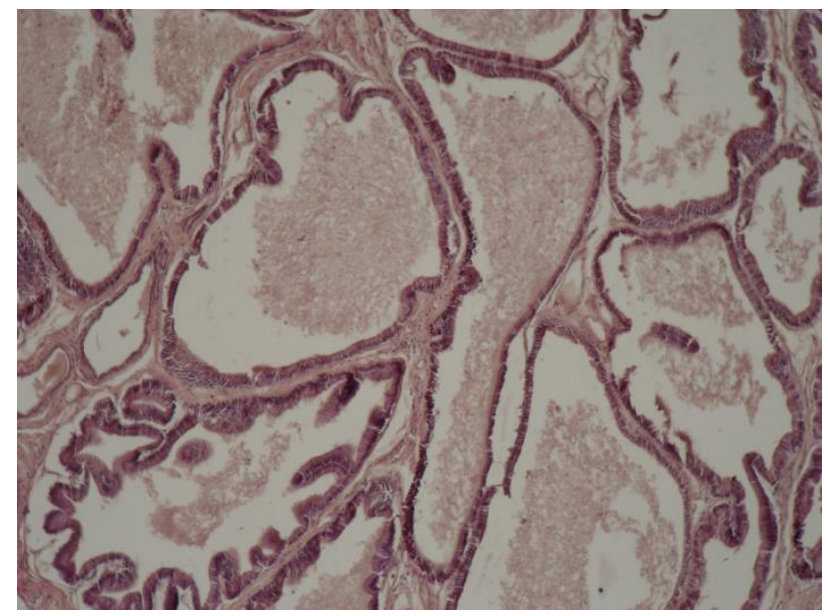

Figure 5. Histological section of prostate from $\mathrm{E}_{3}$ group, Hematoxylin-Eosin stain, 200x a) epithelial desquamation and falling in lumen, b) epithelial segmental necrosis, c) fibrinous exudate

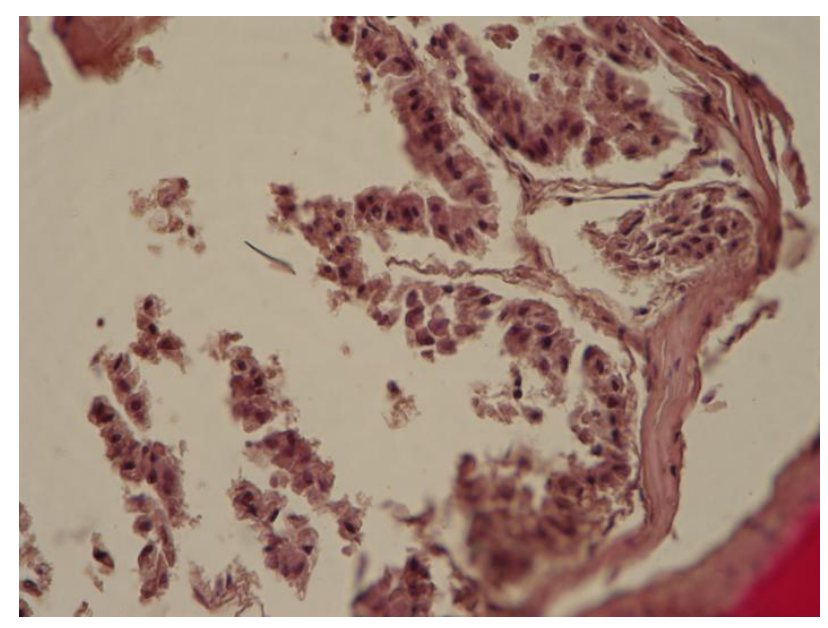

Figure 6. Histological section of seminal vesicles from $\mathrm{E}_{3}$ group, Hematoxylin-Eosin stain, 400x $\leftarrow$ epithelial cells desquamation and falling in lumen

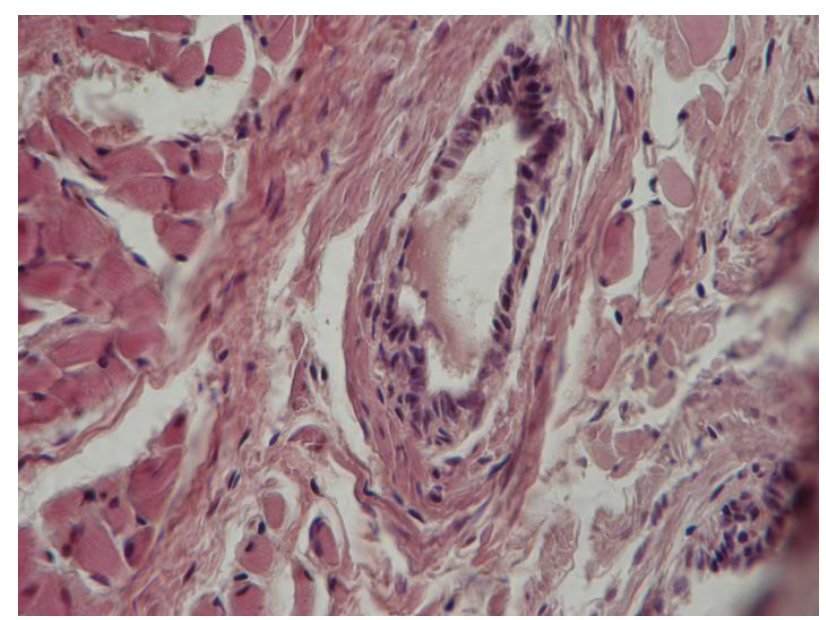

Figure 7. Histological section of bulbo-urethral glands from $\mathrm{E}_{2}$ group, Hematoxylin-Eosin stain, 400x a) flattening of the epithelium, b) epithelial desquamation 
they represent the target organs (Aruldhas et al., $2004,2005)$. As a result of the accumulation of chromium, congestive-necrotic lesions appear in the genital organs and accessory sexual glands. The responsibility of hexavalent chromium for the occurrence of structural changes in the testicles of adult rats has been demonstrated by some researchers (Chandra et al., 2007, 2010; Li et al., 1999).

Hexavalent chromium is repsonsable for reactive oxigen species production, which damages the cell membrane by modifying the fluidity and permeability of its lipid component (Dobrestov et al., 1977), altering the structural integrity of the plasmalemma (Gutteridge and Halliwell, 2000), which may underlie histological changes.

In adult monkeys exposed to hexavalent chromium, presence of cell abnormalities in seminiferous tubules were also described (Aruldhas et al., 2005).

Jana et al. (2006) observed that testosterone is also responsible for the arrangement of the cells in the epithelium of the seminiferous tubes, which due to exposure to hexavalent chromium is disrupted (Chandra et al., 2010).

The decrease of the number, as well as the degeneration of the Leydig cells following the exposure to hexavalent chromium, is also supported by other researchers (Chandra et al., 2010; Trif et al., 2011; Rankov et al., 2010a, 2010b). The decrease of the diameter, but also the hyperplasia of the Leydig cells was also observed (Aruldhas et al., 2005).

Degenerative lesions in testes with disintegration of spermatocytes, atrophy and necrosis of the seminiferous tubes with decreasing diameter, detachment of germ cells from tubular epithelium, lesions present in individuals from experimental groups, coincide with the reports of other authors (Aruldhas et al., 2005; Chandra et al., 2010; Li et al., 1999; Trif et al., 2011; Rankov et al., 2010a, 2010b; Oliveira et al., 2010).

However, Oliveira et al. (2010) did not observe histological changes in the testes and epididymis following administration of $10 \mathrm{mg}$ of potassium chromate / $\mathrm{kg}$ body weight.
Affection of the structure of the prostate and seminal vesicles, respectively the appearance of interstitial edema, necrosis and epithelial exfoliation, are scientifically supported (Trif et al., 2011; Rankov et al., 2010a, 2010b).

In the literature studied there were no references to the occurrence of structural changes in the bulb-urethral glands following exposure to hexavalent chromium compounds.

\section{Conclusion}

Male rat exposure to potassium dichromate (Cr VI) during the suckling period determined severe structural changes in genital organs and sexual accessory glands (congestive and degenerative lesions): interstitial edema, wavy basal membrane due to a reduction in tubule diameter, basal membrane and epithelium degenerative-necrotic lesions, Leydig cell necrosis (testis); interstitial edema, epithelial necrosis and exfoliation, flattening of the epithelium (epididymis); epithelial desquamation and falling in lumen, epithelial segmental necrosis, flattening of the epithelium, fibrinous exudate (prostate, seminal vesicles and bulbo-urethral glands).

Acknowledgments. This research did not receive any specific grant from funding agencies in the public, commercial, or not-for-profit sectors.

\section{References}

1. ATSDR Agency for Toxic Substances and Disease Registry (2012). Toxicological Profile for Chromium, https://www. atsdr.cdc.gov/toxprofiles/tp7.pdf. Accessed 10.07.2019.

2. Aruldhas MM, Subramanian S, Sekar P, Vengatesh G, Chandrahasan G, Govindarajulu P, Akbarsha MA (2005). Chronic chromium exposure-induced changes in testicular histoarchitecture are associated with oxidative stress: study in a non-human primate (Macaca radiate Geoffroy). Human Reproduction, 20(10):2801-2813.

3. Aruldhas MM, Subramanian S, Sekhar P, Hasan GC, Govindarajulu P, Akbarsha MA (2004). Microcanalization in the epididymis to overcome ductal obstruction caused by chronic exposure to chromium - a study in the mature bonnet monkey (Macaca radiata Geoffroy). Reproduction, 128(1):127-137.

4. Chandra AK, Chatterjee A, Ghosh R, Sarkar M (2010). Vitamin E-supplementation protect chromium (VI)induced spermatogenic and steroidogenic disorders in testicular tissue of rats. Food and Chemical Toxicology, 48:972-979.

5. Chandra AK, Chatterjee A, Ghosh R, Sarkar M, Chaube SK (2007). Chromium induced testicular impairment in 
relation to adrenocortical activities in adult albino rats. Reproductive Toxicology, 24:388-396.

6. Directive $2010 / 63 /$ EU of the European Parliament and of the Council of 22 September 2010 on the protection of animals used for scientific purposes, (2018) http://eur-lex.europa.eu/LexUriServ/LexUriServ. do?uri=0J:L:2010:276:00 33:0079:en:PDF. Accessed 20.05.2019.

7. Dobrestov GF, Brochevskaya TA, Valdimirov YA (1977). The increase in phospholipid bilayer rigidity after lipid peroxidation. FEBS Letter, 84:125-128.

8. EPA Environmental Protection Agency (1998). Toxicological Review of Hexavalent Chromium, https:// cfpub.epa.gov/ncea/iris/iris_documents/documents/ toxreviews/0144tr.pdf., Accessed 11.07.2019.

9. Gutteridge JM, Halliwell B (2000). Free radicals and antioxidants in the year 2000: a historical look to the future, Annals of the New York Academy of Sciences. 899:136-147.

10. Jana K, Jana S, Samanta PK (2006). Effects of chronic exposure of sodium arsenite on hypothalamo-pituitarytesticular activities in adult rats: possible an estrogenic mode of action. Reproductive Biology and Endocrinology, 4:9-21.

11. Li H, Chen Q, Li S, Xu Y, Yao W, Chen C (1999). Studies on male reproductive toxicity caused by hexavalent chromium. Chinese Journal of Preventive Medicine, 33(6):351-353.
12. National Research Council, Institute of Laboratory Animal Research (NRC) (2011). Guidefor the Care and Use of Laboratory Animals, Eighth edition, The NationalAcademies Press, Washington, DC, USA,.

13. O’Brien TJ, Ceryak S, Patierno SR (2003). Complexities of chromium carcinogenesis: role of cellular response, repair and recovery mechanism, Mutat Res., 533:3-36.

14. Oliveira H, Spano M, Guevara MA, Santos TM, Santos C, Pereira ML (2010). Evaluation of in vivo reproductive toxicity of potassium chromate in male mice. Experimental and Toxicologic Pathology, 62:391-404.

15. Rankov J, Trif A, Brezovan D (2010b). Six months exposure to potassium dichromate outcomes on histoarchitecture of genital organs and sexual accessory glands in male rats. Lucrări Științifice Medicină Veterinară, Iași, 53, 12(3):509-513.

16. Rankov J, Trif A, Brezovan D, Muselin F (2010a). Potassium dichromate impact on male reproductive integrity biomarker in rat. Two generation study. Scientific Papers Animal Science and Biotechnology, 43(1):224-229.

17. Stoecker B (2004). Chromium, In: Merian, E., Anke, M., Ihnat, M., Stoeppler (Eds.), Elements and their Compounds in the Environment $2^{\text {nd }}$ edition, vol. 2, (pp. 709-729),WILEY - VCN Verlag GmbH \& Co. KGaA.

18. Trif A, Rankov J, Brezovan D (2011). In utero potassium dichromate exposure outcome on biomarker of exposure (chromium level) and integrity (histoarchitecture) of reproductive system in male rat. Lucrari Stiintifice Medicina Veterinara, 44 (2):112-118. 